\title{
Ventriculoperitoneal Shunt without Hair Shaving Using Absorbable Suture Materials
}

\author{
Yun Ho Lee, 'Young Sub Kwon, Jin Mo Cho \\ Department of Neurosurgery, National Health Insurance Service Ilsan Hospital, Goyang, Korea \\ Department of Neurosurgery, ${ }^{2}$ Catholic Kwandong University College of Medicine, Gangneung, Korea
}

Objective : Infection is one of most devastating complications in ventriculoperitoneal (VP) shunt surgery. Preoperative hair removal has traditionally been performed to reduce infectious complications. We performed VP shunt surgeries and evaluated the prevalence of infection in patients who were shaved and those who were unshaven.

Methods : A retrospective analysis was conducted of 82 patients with hydrocephalus of various pathologies who underwent VP shunt surgery, with or without having the head shaved, between March 2010 and March 2017. For patients in the non-shaved group $(n=36)$, absorbable suture materials were used for wound closure, and Nylon sutures or staples were used in the shaved group $(n=46)$. We evaluated the infection outcomes of patients in the two groups.

Results : There was no difference in the average age of patients in the two groups. In the non-shaved group, there were no infections, while two patients in the shaved group required revision because of shunt infection.

Conclusion : Non-shaved shunt surgery may be safe and effective, with no increase of infection rate. We recommend that shunt procedures could be performed without shaving the hair, which may increase patients' satisfaction without increasing infection risk.

Key Words : Ventriculoperitoneal shunt $\cdot$ Hair removal $\cdot$ Infections.

\section{INTRODUCTION}

Infection is one of most devastating complications in ventriculoperitoneal (VP) shunt surgical procedures. Several risk factors of VP shunt infection include age (both younger and elderly patients), previous infection, shunt revision, prolonged operating time, and presence of postoperative cerebrospinal fluid leak ${ }^{6,12,20)}$

Preoperative hair removal, either whole or partly shaving, for cranial neurosurgical procedures has been performed tra- ditionally to reduce infectious complications. Additionally, there are practical reasons for preoperative hair removal, including facilitating access to the incision line, as well as aiding in wound closure and postoperative wound care ${ }^{5,79,10}$. Recently, however, the number of cranial operations with minimal or no preoperative shaving has been gradually increasing ${ }^{11}$, and a number of papers have been published indicating that nonshaving elective cranial surgery does not increase the incidence of infection ${ }^{5,710)}$.

While numerous studies discuss various risk factors of VP

- Received : March 20, 2020 •Revised : April 9, 2020 •Accepted : April 28, 2020

- Address for reprints : Jin Mo Cho

Department of Neurosurgery, Catholic Kwandong University College of Medicine, 24 Beomil-ro 579beon-gil, Gangneung 25601, Korea

Tel : +82-32-290-3182, Fax : +82-32-290-3187, E-mail : ns9@naver.com, ORCID : https://orcid.org/0000-0002-1192-8993

This is an Open Access article distributed under the terms of the Creative Commons Attribution Non-Commercial License (http://creativecommons.org/licenses/by-nc/4.0) which permits unrestricted non-commercial use, distribution, and reproduction in any medium, provided the original work is properly cited. 
shunt infection, none discuss the role of preoperative shaving. In a few studies that have performed non-shaved VP shunt procedures, they did not report an increase in VP shunt infections $^{2,3)}$. However, more research is still needed to determine the efficacy of performing shunt surgery without preoperative shaving.

Since 2010, we have been performing non-shaved cranial surgeries for various necessary implants or catheterizations, such as VP shunts and brain stimulations. Given the growing evidence that non-shaved procedures may not be associated with increased infection risk, we evaluated the incidence of infections between shaved and non-shaved patients who received VP shunt procedures.

\section{MATERIALS AND METHODS}

\section{Patients}

This study was approved by the Institutional Review Board of Ilsan Hospital (NHIMC 201607003 003) which waived the informed consent due to the retrospective nature of the study. A retrospective analysis was conducted of patients with hydrocephalus of various pathologies who underwent a VP shunt surgical procedure between March of 2010 and March of 2017. A total of $82 \mathrm{VP}$ shunt procedures were performed by single neurosurgeon during this period. The patients were divided into two groups, hair shaved and non-shaved, based on preoperative hair preparation. The shaved group included a total of 46 patients who refused the non-shaving technique. The remaining 36 patients received a VP shunt without preoperative shaving.

\section{Procedure}

The patients in the shaved group had their hair removed using clippers and a razor the morning of the surgery. Nonshaved patients used a routine commercial shampoo the morning or night before the day of surgery. Their hair was parted with a fine-tooth comb and was held away from the surgical site by using rubber bands. In order to prevent hair from entering into the incision site, some hair was fixed with staplers at surgical sterile drape (Fig. 1). All patients typically received intravenous antibiotics (cefotetan, a 2nd generation cephalosporin) prophylactically, as well as for 2 days post operation if there were no complications or issues.

For patients in the non-shaved group, absorbable suture materials (Vicryl Rapide 3-0; Ethicon, Somerville, NJ, USA) were used for wound closure, while Nylon or staples were used in the shaved group. When using the absorbable suture materials, the wound was closed with running or subcutaneous suture techniques to approximate the scalp edges. The entire op-
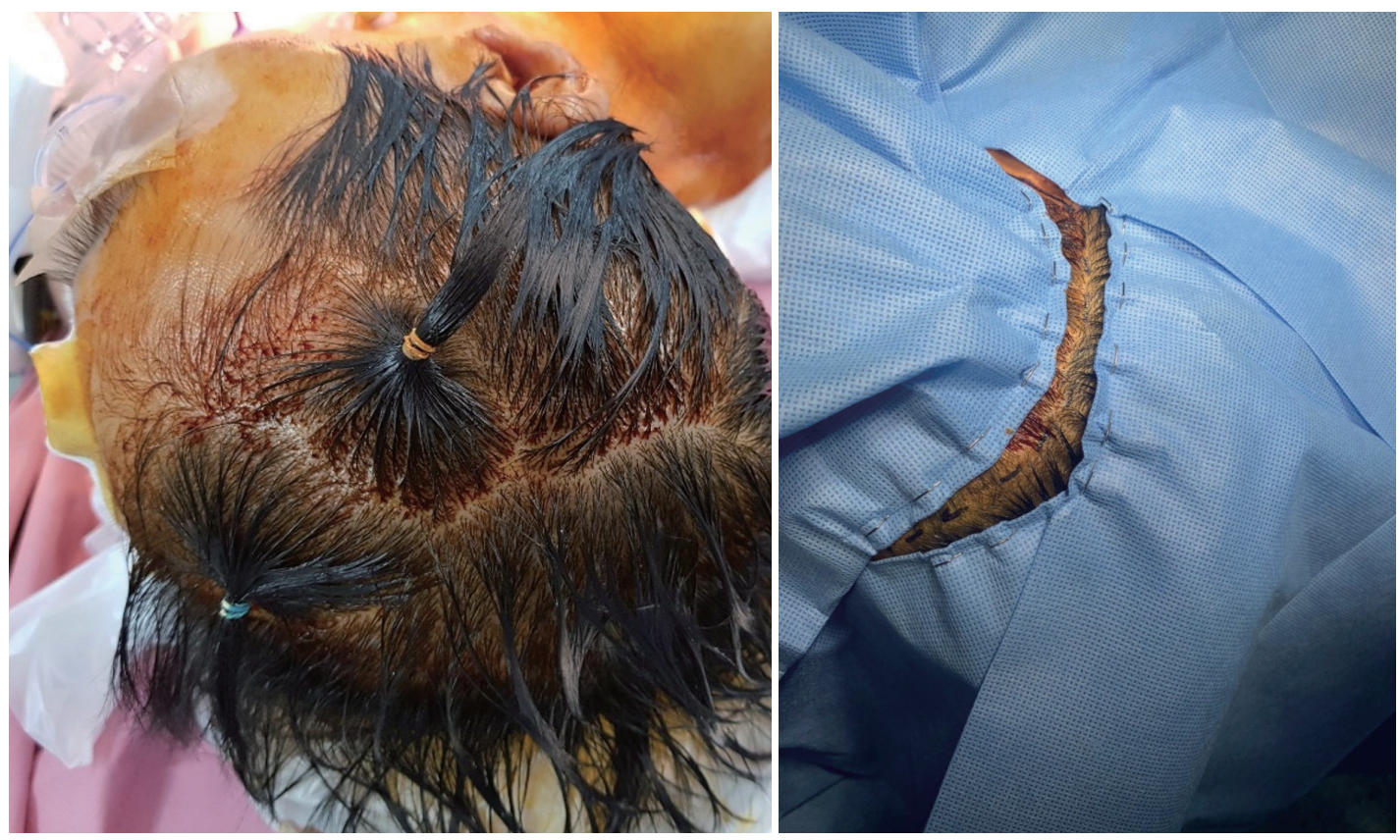

Fig. 1. Hair was parted along the proposed incision line (dashed line) with a stapler and secured with elastic bands. 
erative field was scrubbed with povidone-iodine soap, and then painted with a povidone-iodine solution or soaked with a mixed solution of 75\% alcohol and povidone-iodine solution. The incidence of surgical infection, as well as age and gender of the two groups, was compared and analyzed using chi-squared tests

\section{RESULTS}

We evaluated 36 patients who underwent non-shaved VP shunt surgery using absorbable sutures for scalp closure and 46 patients who underwent shaved VP shunt surgery using non-absorbable scalp sutures. The age of non-shaving group was in the range of 16 to 88 years with a mean of 62.2 years. The sex was 22 males and 14 females. The age of shaving group was in the range 36 to 84 years with mean 64.9 years and there were 26 males and 20 females. The two groups were comparable with respect to general demographics, as they had similar numbers of males and females, age, previous history of surgery and past history such as DM (Table 1).

While there were no infections in the non-shaved group, two patients in the shaved group required revision because of shunt infection. During the 2 -week postoperative period, these two patients had a constant fever and their cerebrospinal fluid culture was positive for Staphylococcus aureus. However, there was no statistical difference $(p>0.05)$ in infection rates between the two groups.

\section{DISCUSSION}

Univariate and multivariate analysis did not show any sig- nificant difference in the shunt infection rate between the shaved and non-shaved groups. In fact, there were two cases of infection in the hair removed group suggesting that hair removal may be related with an infection rate increase, though more studies are needed to investigate this further.

\section{Hair removal and infection}

Shaving of the whole head has been common practice in neurosurgical procedures since the days of Harvey Cushing $^{2,3,6,10,12,20)}$. Shaving allowed for better exposure of the incision line, as well as ease of closure and postoperative wound care, and was known to decrease postoperative infection rate. Although there are pros and cons of scalp shaving in neurosurgery, many neurosurgeons still prefer to practice whole head shaving for cranial surgeries due to the reasons listed. Recently, non-shaved neurosurgery has been the focus of much attention because of quality of life. Many authors reported that shaving of the hair was not related to an increase in infection rates for cranial surgery ${ }^{5,7,9,13,16,18)}$. Bekar et al. ${ }^{4)}$ retrospectively reviewed and compared the rate of infection of 1038 cranial procedures without hair removal and 980 cranial surgeries with hair removal. They reported that there was no significant difference in the rate of infection between patients in each group and concluded that hair removal is not necessary in preparation for any type of cranial surgery. Tanner et al. ${ }^{19)}$ performed a meta-analysis of 11 randomized clinical trials (total of 5031 people) and concluded that there was no evidence that removing hair decreased infection rate. Furthermore, they recommend that if necessary, clipping and using depilatory creams result in fewer surgical site infections than shaving using a razor.

Table 1. Characteristics of patients

\begin{tabular}{lccc}
\hline & Shaved $(\mathbf{n = 4 6 )}$ & Non-shaved $(\mathbf{n = 3 6 )}$ & \multicolumn{1}{c}{-value } \\
\hline Age (years) & $64.9(36-84)$ & $62.2(16-88)$ & 0.981 \\
Sex, F : M & $20: 26$ & $14: 22$ & 0.675 \\
Surgical infection & 2 & 0.501 \\
Reason of VP shunt & $\begin{array}{l}\text { Post hemorrhagic hydrocephalus 41 (89.1) } \\
\text { Obstructive hydrocephalus 2 (4.3) }\end{array}$ & NPH 18 (50.0) & Post hemorrhagic hydrocephalus 11 (30.6) \\
& Others 3 (6.5) & $\begin{array}{l}\text { Congenital hydrocephalus 4 (11.1) } \\
\text { Obstructive hydrocephalus 3 (8.3) }\end{array}$ \\
\hline
\end{tabular}

Values are presented as mean \pm standard deviation unless otherwise indicated. VP : ventriculoperitoneal, NPH : normal pressure hydrocephalus 


\section{Shunt infection}

Although many surgeons know that hair removal may not be related to decreased risk of infection, they hesitate to perform the surgery unshaved because infection is one of the most concerning surgical complications, particularly in shunt surgery. Infections resulting from many neurosurgical procedures can be cured with antibiotic administration, however, most shunt infection cases need to be treated with shunt removal, repeated extraventricular drainage, and prolonged antibiotic use $\mathrm{s}^{8,15,17)}$. Therefore, many neurosurgeons are reluctant to perform a non-shaved surgery during a shunt procedure. It should be noted that most shunt infections were diagnosed within 1-2 months after surgery, indicating that contamination likely occurred during the shunt surgery itself ${ }^{11,21)}$. All non-shaved patients in our study were followed for more than 6 months without incident of infection. Thus, we believe that hair removal is not necessary for the purpose of reducing the risk of infection.

\section{Advantages of this study and recommendations}

From the surgeon's point of view, these results may provide the evidence needed to demonstrate the efficacy of not shaving in preparation for shunt surgery. From the patient's point of view, having a non-shaved surgery increases their quality of life as they are able to return to their previous social life earlier than if their head had been shaved ${ }^{14)}$. Furthermore, our results may support the practice guideline that scalp shaving could increase the rate of wound infection; therefore, the practice should be abandoned.

We spent approximately 5 to 10 minutes more during preparation to manage the hair for patients who did not have their head shaved than for patients who had their hair removed. However, this additional preparation time is not related with increased infection risk as this is done prior to the procedure itself and there is no open wound. For hospitals and institutes who implement this procedure, proper protocols should be developed that aim to minimize this time for hair preparation. When we first began performing non-shaved surgeries, we would try to split the hair completely to clear it from the incision site, and in some cases, we would remove hair if it interfered with the sutures. However, over time, we found that there were no issues if some of the hairs were caught in the sutures.

\section{Limitation}

This study has a few limitations. First, this was a retrospective comparative study of two surgical policies for a single surgeon. Second, our study had a relatively small number of patients, which is a limitation for some statistical analyses. Therefore, larger, controlled case studies should be conducted before generalizing these results.

\section{CONCLUSION}

Our results provide evidence that non-shaved shunt surgery is safe and effective, and likely increases patients' quality of life and satisfaction. We recommend that shunt procedures could be performed without hair shaving as it may increase patients' satisfaction without increasing infection risk.

\section{CONFLICTS OF INTEREST}

No potential conflict of interest relevant to this article was reported.

\section{INFORMED CONSENT}

This type of study does not require informed consent.

\section{AUTHOR CONTRIBUTIONS}

\author{
Conceptualization : JMC, YHL \\ Data curation : YHL, YSK \\ Formal analysis : JMC \\ Methodology : JMC, YHL \\ Project administration : JMC, YSK \\ Visualization : JMC \\ Writing - original draft : JMC \\ Writing - review \& editing : JMC
}

\section{ORCID}

Yun Ho Lee https://orcid.org/0000-0001-8156-5414 
Young Sub Kwon https://orcid.org/0000-0002-2996-2145

Jin Mo Cho

\section{References}

1. Adeleye AO : Nonshaved cranial surgery in black Africans: technical report and a medium-term prospective outcome study. Neurosurg Rev 39 : 449-454, 2016

2. Albright AL, Pollack IF, Adelson PD : Principles and practice of pediatric neurosurgery. New York : Thieme, 1999

3. Alexander JW, Fischer JE, Boyajian M, Palmquist J, Morris MJ : The influence of hair-removal methods on wound infections. Arch Surg 118 : 347-352, 1983

4. Bekar A, Korfalı E, Doğan Ş, Yılmazlar S, Başkan Z, Aksoy K : The effect of hair on infection after cranial surgery. Acta Neurochir (Wien) 143 : 533-536; discussion 537, 2001

5. Bhatti MI, Leach PA : The incidence of infection for adults undergoing supra-tentorial craniotomy for tumours without hair removal. Br J Neurosurg $27: 218-220,2013$

6. Braun $\mathrm{V}$, Richter $\mathrm{H}$ : Shaving the hair--is it always necessary for cranial neurosurgical procedures? Acta Neurochir (Wien) 135 : 84-86, 1995

7. Chesnut RM, Temkin N, Carney N, Dikmen S, Rondina C, Videtta W, et al. : A trial of intracranial-pressure monitoring in traumatic brain injury. N Engl J Med 367 : 2471-2481, 2012

8. Conen A, Walti LN, Merlo A, Fluckiger U, Battegay M, Trampuz A : Characteristics and treatment outcome of cerebrospinal fluid shuntassociated infections in adults: a retrospective analysis over an 11-year period. Clin Infect Dis 47 : 73-82, 2008

9. Iwami K, Takagi T, Arima T, Takayasu M : Cranial surgery without shaving: practice and results in our hospital. No Shinkei Geka 34 : 901-

905, 2006

10. Ko W, Lazenby WD, Zelano JA, Isom OW, Krieger KH : Effects of shaving methods and intraoperative irrigation on suppurative mediastinitis after bypass operations. Ann Thorac Surg 53 : 301-305, 1992

11. Lo P, Drake JM : Shunt malfunctions. Neurosurg Clin N Am 12 : 695701, viii, 2001

12. Orsi GB, Ferraro F, Franchi $C$ : Preoperative hair removal review. Ann Ig $17: 401-412,2004$

13. Ratanalert S, Musikawat $P$, Oearsakul T, Saeheng S, Chowchuvech V: Non-shaved ventriculoperitoneal shunt in Thailand. J Clin Neurosci 12 : 147-149, 2005

14. Ratanalert S, Saehaeng S, Sripairojkul B, Liewchanpattana K, Phuenpathom N : Nonshaved cranial neurosurgery. Surg Neurol 51 : 458463, 1999

15. Ratanalert $\mathrm{S}$, Sriplung $\mathrm{H}$ : Social attitudes toward shaving for cranial neurosurgery. Br J Neurosurg 15 : 132-136, 2001

16. Sarmey N, Kshettry VR, Shriver MF, Habboub G, Machado AG, Weil RJ : Evidence-based interventions to reduce shunt infections: a systematic review. Childs Nerv Syst 31 : 541-549, 2015

17. Schreffler RT, Schreffler AJ, Wittler RR : Treatment of cerebrospinal fluid shunt infections: a decision analysis. Pediatr Infect Dis J 21 : 632636, 2002

18. Tanner J, Moncaster K, Woodings D : Preoperative hair removal: a systematic review. J Perioper Pract 17 : 118-121, 124-132, 2007

19. Tanner J, Woodings D, Moncaster K : Preoperative hair removal to reduce surgical site infection. Cochrane Database Syst Rev (3) : CD004122, 2006.

20. Winston KR : Hair and neurosurgery. Neurosurgery 31 : 320-329, 1992

21. Wu X, Liu Q, Jiang X, Zhang T : Prevention options for ventriculoperitoneal shunt infections: a retrospective analysis during a five-year period. Int J Clin Exp Med 8 : 19775-19780, 2015 